Chapter 5

\title{
The Role of Id 2 in the Regulation of Chromatin Structure and Gene Expression
}

\author{
Elena R. García-Trevijano, Luis Torres, \\ Rosa Zaragozá and Juan R. Viña \\ Additional information is available at the end of the chapter \\ http://dx.doi.org/10.5772/54969
}

\section{Introduction}

\subsection{The helix-loop-helix Id2 proteins}

Cell function and tissue homeostasis are dependent on the precise regulation of multiple and converging pathways that ultimately will control a variety of biological processes such as cell proliferation, growth arrest, differentiation or apoptosis. Frequently these pathways share one or several steps in the signal cascade resulting in either redundant or opposing responses.

Chromatin structure is an essential part of the regulatory mechanisms for gene transcription. Moreover, chromatin has been revealed as a dynamic structure exquisitely regulated through multiple mechanisms including histone modifications and chromatin remodeling complexes that finally, will give rise to an open or closed structure changing the accessibility of specific transcription factors to nucleosomal DNA. Therefore, the protein complexes involved in the modulation of chromatin remodelers and histone acetyltransferases (HAT) or deacetylases (HDAC) could be somehow considered as part of a previous sensitization process necessary for a full and specific biological response.

In the recent literature there is a number of papers describing a variety of biological processes in which cells from different tissues need to be previously sensitized or "primed" to achieve a full response to either a chemical or a biological signal. This concept has been extensively studied in the experimental model of liver regeneration after 2/3 partial hepatectomy ( $\mathrm{PHx}$ ). Hepatocytes in normal liver are quiescent, resting at the G0 phase of the cell cycle, and exhibit a poor response to potent in vitro mitogens. Once hepatocytes reach the early G1 phase, cells respond to those mitogens and can progress through the cell cycle. Consequently, it is generally accepted that hepatocytes need to be previously sensitized to overcome the G1 restriction point 
and become competent for DNA replication [1]. On the other hand, it has been long known that apoptosis-inducing cytokines such as TNF- $\alpha$ are not able to trigger apoptosis in hepatocytes unless they are accompanied by chemicals such as actinomycin D or cycloheximide [2].

Accordingly, unveiling the nature of those signals participating in the cell priming events would be crucial to understand the molecular mechanisms mediating, not only the physiology of different biological processes, but also the pathogenesis of many diseases. We propose here that the helix-loop-helix (HLH) protein Id2 has an important role in the process of cell sensitization by a mechanism affecting chromatin structure of selected genes.

Id2 belongs to a family of HLH proteins identified and named over two decades ago for its dual role as both, inhibitors of the differentiation process and inhibitors of DNA binding. Id proteins were initially found during a screen for determination of factors from the family of basic-helix-loop-helix (bHLH) proteins. Paradoxically, Id proteins lack the basic region adjacent to the HLH domain [3]. This region rich in basic aminoacids, has been shown to be essential for specific binding to DNA: It binds to a hexanucleotide E box sequence on the DNA of target genes (CANNTG) [4].

The HLH proteins are classified into seven groups of factors according to their DNA-binding motif. The seven classes of HLH proteins share highly conserved amphipatic helices connected by a loop of variable sequence and length.

Class-I (or class-A) bHLH proteins are also known as the E proteins since they are encoded by spliced variants from the EA2 gene. This group includes proteins that are ubiquitously expressed. Class-II (or class-B) bHLH proteins, includes members such as MyoD, NeuroD, or Hes with a tissue-restricted pattern of gene expression. Class-I and class-II proteins can form either homo- or heterodimers. Binding of these dimers to "E box" in the promoters of tissuespecific genes will regulate a number of developmental processes [for review see reference 5]. Id proteins belong to the class-V of HLH factors including four members going from Id1 to Id4 [3-6]. Id proteins can dimerize with either class-I or class-II proteins but since they do not contain the DNA-binding motif, this dimerization will inhibit class-I and class-II factor-activity in a dominant-negative fashion [3]. Consequently, the developmental processes modulated by Class-I and-II factors will be blocked by Id proteins [6-10]

Over the past few years among all members of Id family of proteins, Id2 has been shown to be essential for the modulation of biological processes other than its first described function during cell differentiation and development. Among these functions, Id 2 has been found to be involved in the regulation or coordination of cell proliferation, cell cycle control, senescence, apoptosis or angiogenesis and metastasis [6,11-16]. Moreover, data in the literature describing the role of Id 2 in different cell types suggest that Id 2 function is highly dependent on the cell microenvironment and that its molecular mechanism of action is far more complicated than it was thought at first. Since the expression of individual genes is induced in response to a variety of stimuli and frequently, the same signal participates in so many different biological processes, the study of all Id 2 functions turns out to be extremely complex. In this review we discuss some of the potential mechanisms by which Id2 has been proposed to modulate cell prolifer- 
ation under both, physiological and pathological conditions. We also present the newest findings of the signaling network leading to the modulation of Id2 activity

\section{Role of Id2 in cell proliferation}

In addition to other functions described for Id2, it seems that its role as a proliferative factor is now recognized as one of the most significant. In fact, there is a growing number of evidences derived from both, in vitro and in vivo experiments in support of this notion. Ectopic overexpression of Id 2 in different cell types enhances proliferation $[17,18]$ and cells acquire some features of transformation [19]. More importantly, in some human cancers such as pancreatic, neuroblastoma or colon carcinoma among others, Id2 is over-expressed [20-22]. Partial ablation of Id2 by antisense oligonucleotides in these tumors decreases cell proliferation. Indeed, Id2 has been proposed as a prognostic factor and a potential therapeutical target for cancer treatment $[15,20,23]$.

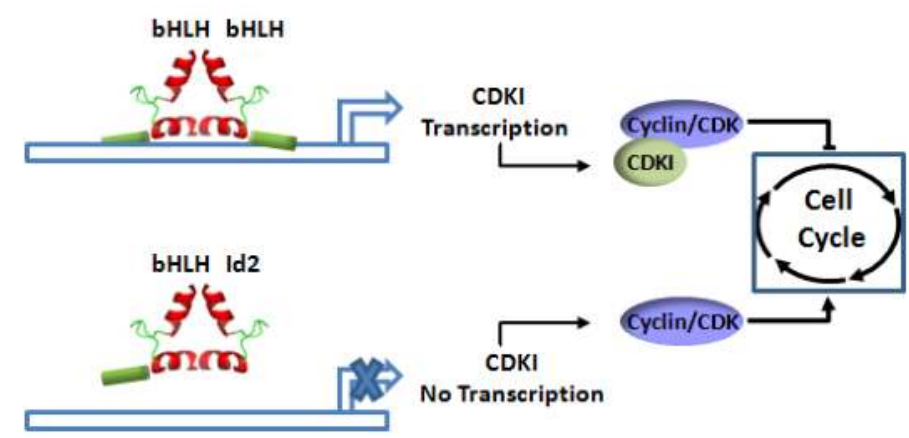

Figure 1. Model of Id2 as a dominant-negative factor for the cell cycle control. (Upper) Proteins of the bHLH family dimerize and bind to the promoter of cdki genes by their basic domain. Transcription of cdki is up-regulated. CDKI binds to the cyclin/CDK complex and blocks its activity. Cell cycle will be blocked. (Lower) bHLH proteins dimerize with Id2. Since Id 2 lacks a basic domain, the dimer bHLH/Id2 cannot bind to gene promoters. CDKI transcription is downregulated. Cyclin/CDK complex is active and cell cycle will progress.

\subsection{Molecular mechanisms}

From the classical point of view, the role of Id2 as a proliferative factor was thought to be dependent only on its ability to dimerize with bHLH proteins as a dominant-negative factor (Figure 1). In this model, Id 2 would control cell proliferation by repressing the expression of cell cycle inhibitors such as $\mathrm{p}^{\mathrm{Cip} 1}$ or p57 ${ }^{\mathrm{Kip} 2}$ [24-26]. Nevertheless, later was demonstrated that $\mathrm{Id} 2$ could bind to proteins different from $\mathrm{bHLH}$ factors such as the tumor suppressor $\mathrm{pRB}$ and the pRb-related proteins p130 and p107 [20]. In this sense, it is largely known that RB (referred to $\mathrm{pRb}$ or the pocket proteins) directly associates with E2F on E2F-responsive promoters to restrain gene expression of cell cycle-related genes [ for review see reference 27]. Therefore, these data ultimately connect Id2 and E2F-target genes. 
E2Fs contribution to cell proliferation and the molecular signaling network that converges on $\mathrm{RB}$ proteins has been extensively documented. Although all RB proteins bind to E2F and are modulated by a cell-cycle dependent mechanism, their pattern of expression is different. Whereas p130 is most prominent in arrested cells and preferentially binds to E2F4/5, p107 is mainly expressed in proliferating cells. Finally, pRB is expressed in both proliferating and quiescent cells. The phosphorylation of RB proteins by specific cyclin/CDK complexes will render the subsequent waves of E2F-dependent transcription that will guarantee the sequential transition of cells through the cell cycle (figure 2):

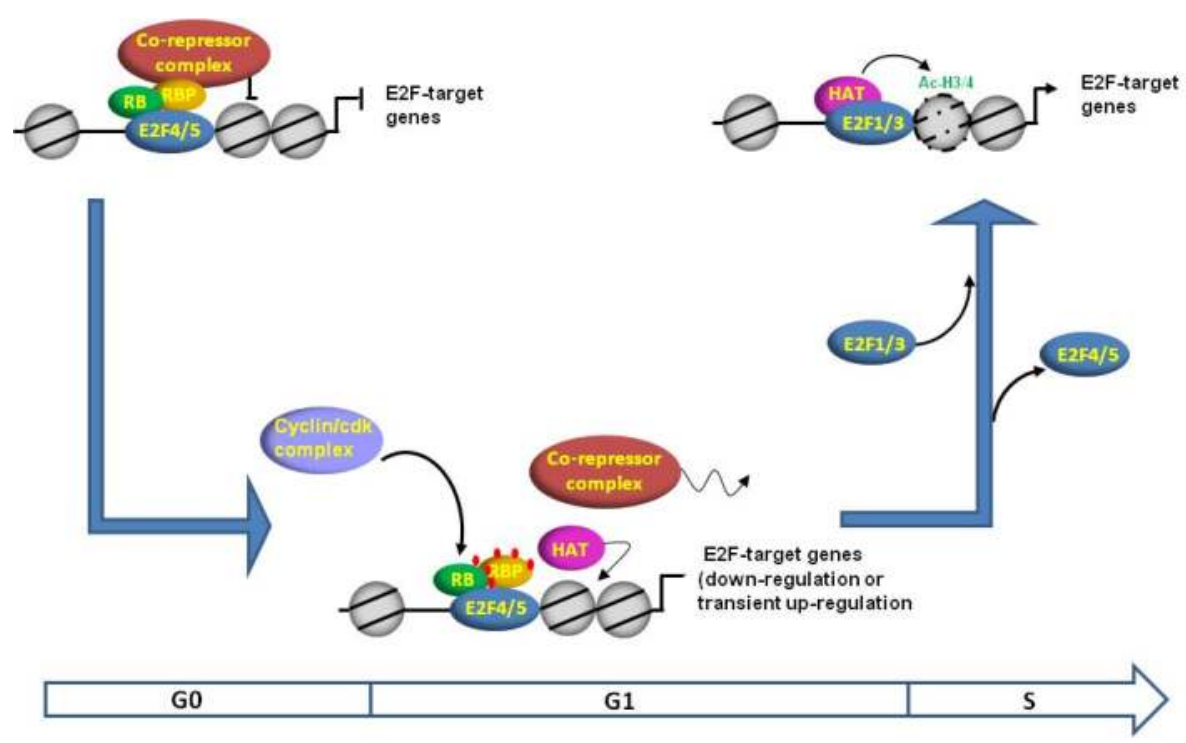

Figure 2. Cell cycle control of E2F-target genes. In G0 cells, E2F4/5 associates with RB proteins and RBP. RBP connects $\mathrm{RB}$ to co-repressors (HDAC). E2F-target genes are repressed at this phase. Upon stimulation, sequential phosphorylation of both, RB and RBP will release the co-repressors and transition G0-G1 will take place. HATs could have access to chromatin as G1 progress. At late G1 phase cumulative phosphorylation of RB and RBP will result in the loss of RB function, release of E2F4/5 and the accumulation of newly synthesized E2F1,-2 and -3. These events will drive cells into the $S$ phase.

In quiescent or differentiated cells, ubiquitously expressed E2F4/5 and p130 can bind simultaneously to various co-repressors such as DNA methyltransferase 1 (DNMT1) or histone deacetylases (HDAC) complexes. p130 will inhibit E2F4/5 transcriptional activity by two mechanisms, including binding and inhibition of the E2F transactivation domain, and by recruiting co-repressors such as those mentioned above. These events will lead to chromatin compaction and transcriptional inhibition of genes necessary to entry into the $S$ phase of the cell cycle.

Upon a mitogenic stimulus, during G1 phase, phosphorylation of RB binding proteins (RBP) by cyclinD/CDK4/6 and cyclin E/A/CDK2 will dissociate the transcriptional co-repressors from E2F allowing the access of acetyltransferases (HATs) to gene promoters [28]. 
At the late G1 phase of the cell cycle, the concurrent and cumulative CDK-mediated phosphorylation of $\mathrm{pRb}$ and/or p107 will result in the loss of RB function.

Finally, during G1 to S phase transition, newly synthesized E2F1, -2 and -3 will substitute E2F4/5 and, HATs will be recruited to gene promoters. These two events will drive cells into the $S$ phase.

In this context Id2 seems to take part in a crucial checkpoint for cell proliferation since it can bind to each of the pocket proteins of the RB family in a cell cycle-regulated fashion [20]. Importantly, Id 2 is the only member of Id family of proteins that can bind to hypophosphorylated pRB, p107 or p130 [26]. Furthermore, Id2 has been shown to disrupt the growth suppressive activity of RB when the latter is unphosphorylated $[17,20,26]$.

The observation of the pattern of Id2 expression following a mitogenic stimulus suggests different functional roles for this protein during cell cycle progression. The first peak of Id2 expression takes place during the transition from G0-G1[13]. Id2 could have a role in the modulation of p130-E2F4/5 complex activity and in the priming events that occur during this reversible phase of the cell cycle. The second peak of Id2 expression takes place at late G1 phase, where Id 2 could disrupt the repressor activity of p107 and/or pRb facilitating the entry into the $\mathrm{S}$ phase. The link between Id2 and RB has been further confirmed in animal experiments with double knockout mice for both RB and Id2 [20]. The early lethal phenotype shown in $\mathrm{Rb}$-/- embryos was rescued by Id 2 ablation. Several mechanisms not mutually exclusive, have been proposed to explain the physiological role of Id2 on the RB pathway (figure 3):

1. Id2 binding to tumor suppressor protein RB would interfere with its anti-proliferative functions. Expression of Id2 is induced as a response to mitogenic stimuli in a variety of cell types. Therefore, a stoichiometric excess of Id 2 would block RB by direct binding to this tumor suppressor $[9,12,29]$. E2F is an important factor involved in RB function. Although the direct binding of Id2 to RB proteins have been demonstrated, the relationship between cellular RB-E2F and RB-Id2 complexes is poorly understood. Immunoprecipitation experiments have shown that E2F, RB and Id2 take part of the same complex in rat liver, most probably bound to gene promoters [30]. These data suggests that Id 2 and E2F can bind simultaneously to the same molecule of RB (figure 3a). Nevertheless, it cannot be exclude the possibility that Id 2 and E2F would compete for binding to RB (figure $3 b)$. In this model, the cellular excess of pocket proteins is the main safeguard mechanism of negative control on cell cycle progression.

2. In addition to RB, the targets of Id 2 could be the effectors of RB-mediated cell cycle arrest (figure 3c). These effectors could be either, bHLH or non-bHLH proteins that can also modulate the expression or activity of important proteins controlling the cell-cycle.

CDK inhibitors (CDKIs) enhance the suppressor activity of RB proteins blocking cell cycle progression. Therefore, proteins involved in the modulation of CDKI expression or activity could be considered as part of the RB effectors. As mentioned above, Id 2 blocks the expression of cell cycle inhibitors such as $\mathrm{p} 21^{\mathrm{Cip} 1}$ or $\mathrm{p} 57^{\mathrm{Kip} 2}$. Over-expression of Id 2 was able to reverse

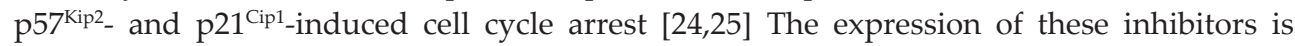


modulated by bHLH transcription factors. Id2 heterodimerization with these factors will lead to CDKI repression and activation of CDKs. Moreover, MyoD a class II

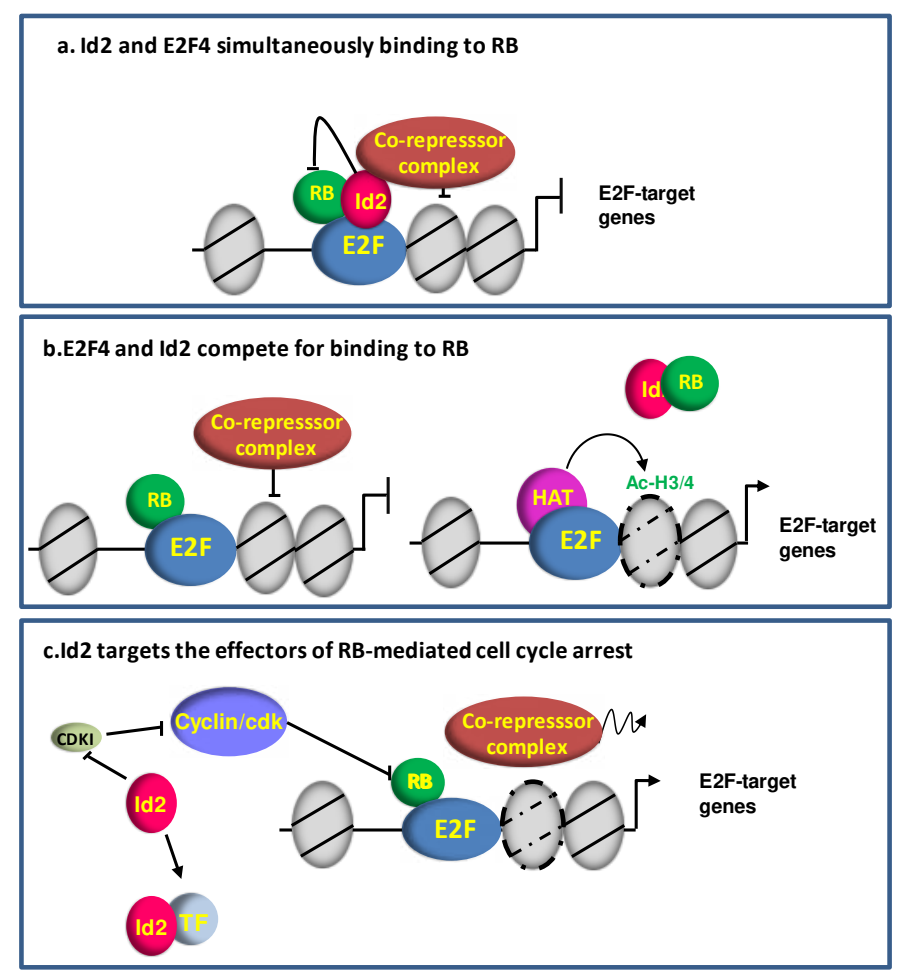

Figure 3. Models for Id 2 function associated to RB proteins.(a) Id 2 and E2F4 simultaneously bind to the same molecule of RB on gene promoters. Id 2 excess gets restrain the repressive activity of RB. (b).E2F4 and Id 2 compete for binding to RB. When RB is in excess prevents binding of Id 2 to E2F and keeps its repressive activity. An excess of Id 2 prevents binding of RB to E2F and restrains its repressive activity recruiting HATs to gene promoters. (c) Id 2 targets the effectors of RB-mediated cell cycle arrest. Id 2 dimerization with bHLH factors down-regulates the expression of CDKIs. RB hyperphosphorylation by free cyclin/CDKs will lead to the transcription of E2F-target genes. In addition, Id2 dimerization with non-bHLH transcription factors will block their DNA-binding activity necessary to modulate transcription of E2F-target genes.

bHLH protein known to induce $21^{\text {Cip1 }}$ transcription, has been shown to act by itself as a CDKI. MyoD binds to and inhibits CDK4 [31]. Therefore, Id2 heterodimerization with MyoD would block MyoD function as a CDKI.

In addition to bHLH proteins, Id2 (and other members of the Id family of proteins) also binds to other classes of transcription factors. A brief example of these non-bHLH factors includes Pax [32] or factors from the ternary complex (TCF) sub-family of ETS-domain proteins. TCF proteins such as Elk-1, SAP-1 and SAP-2 modulate the expression of immediate-early genes following a mitogenic stimulus by binding to serum response factor (SRF). Id 2 binding to the 
ETS DNA binding domain of TCFs prevents the interaction TCF/SRF and disrupts binding of this complex to the serum responsive element on gene promoters [33].

In all these models, Id2 activity could be restrained by RB to keep its anti-proliferative function as shown by Lasorella [20]. The negative role of RB proteins on Id 2 could be essential to keep cell cycle arrest for two reasons: First RB would be free to bind to and to restrain the expression of E2F-target genes, and second it would prevent Id2 binding to other targets. On the other hand, a high concentration of Id2, as it occurs in neoplastic transformation, may relieve E2Fdriven transcription from the repressive influence of RB. In addition, Id2 would enhance cell proliferation modulating the expression and/or activity of other targets.

\subsection{Id 2 in cancer: Increased cell proliferation and survival}

The role of Id proteins in cancer has been of much interest in the last decade. Variations in expression levels of Id proteins have to be integrated into the whole cellular equilibrium. An upstream deregulation of Id activity, going from Id gene transcription to Id protein, can result in the downstream deregulation of diverse genes. The first evidence connecting Id2 expression and cancer came from the observation that Id2 is a transcriptional target of both N-Myc and c-Myc [20]. Id 2 transcription is induced by Myc via binding to E-boxes on Id2 promoter. It has been suggested that Myc increases Id 2 expression to bypass the inhibitory activity of RB. TGF$\beta$, a cytokine known to induce a cytostatic program, inhibits Id2 expression in epithelial cells and in human keratinocytes by induction of the Myc antagonistic repressors Mad2 and Mad4. Replacement of Myc-Max complexes with Mad-Max complexes on the Id2 promoter drives Id2 downregulation [34].

Additional evidence for the oncogenic role of Id2 came from studies in which ectopic overexpression of Id2 in developing thymocytes caused a rapid development of lymphomas [35]. On the other hand, aberrant Id2 expression has been reported in squamous cell carcinoma, primary human colorectal adenocarcinomas, pancreatic and prostate cancer or neuroblastoma [11-16]. Id 2 has also been related to cancer progression, showing that Id 2 expression was higher in high-grade astrocytic tumors than in low-grade tumors [36].

Data in the literature point out to the transcriptional control of Id2 as a key event in the modulation of its function. In this sense, a highly expressed Id2 is not only involved in cell proliferation, but it also promotes cell survival in different cell types. Over-expression of Id2 blocks the TGF- $\beta$-induced apoptosis [37]. The role for Id 2 as a survival factor in mammary gland development has also been demonstrated [38]. On the other hand, IGF-I known to be a survival growth factor, induces Id2 expression in murine hematopoietic cells [39]. Furthermore, knockdown of Id1 and Id2 gene expression have been shown to induce apoptosis in gut epithelial cells [37]. These observations, together with the fact that ectopic over-expression of Id2 enhances cell proliferation, would suggest that a highly expressed Id2 could confer a proliferative advantage to tumor cells through the modulation of both, growth and survival pathways.

Although it is generally accepted that deregulated expression of Id2 maintains a highly proliferative state and/or extends the half-life of cells in culture [13], Id2 over-expression is not 
sufficient for cell transformation. Actually, we must be careful to distinguish between events in cell culture and in animals, because the requirements for the modulation and role of Id2 in culture may be mainly related to its requirements in a transformed state more than the requirements in a normal physiological situation. Finally, even though Id2 over-expression has been detected in the above mentioned cancer types and its oncogenic activities cannot be denied, its deregulation is relatively rare. In contrast, Id1 over-expression has been detected in a larger number of cancer types [23] and seems to have a more prominent role during cancer progression.

\subsection{Id2 during G0-G1 transition}

Since Id 2 appears to facilitate and not to support cell cycle progression, it is possible that Id2 could have a role in the process of cell priming, sensitizing cells to a proliferative stimulus. This priming events take place during the G0 to G1 transition and, as already mentioned, are needed to obtain a fully proliferative response to mitogenic stimuli. The pattern of Id2 expression during the priming events support this hypothesis: During G0-G1 transition there is a transient increase of Id2 mRNA levels [13,30].

Liver regeneration after $\mathrm{PHx}$ is a unique model to study cell cycle events that take place in a synchronized manner in vivo. The protein c-Myc, proposed as part of the priming events in liver regeneration, modulates the expression of proteins important to progress into the cell cycle. The expression of very-early genes such as $c-f o s, c-j u n$, or $c-m y c$ takes places in a coordinated and sequential way. The up-regulation of some genes will trigger the next wave of gene expression. c-Myc was suggested to use Id2 as a mediator to bypass the suppressive activity of RB $[19,20]$. During G0-G1 transition after PHx in rat liver there is a concomitant and short up-regulation of both Id2 and c-myc mRNA.

In the last decades, c-Myc has been the object of intense study in an attempt to unveil the molecular mechanism behind the control of its abundance [review in ref 40]. c-Myc levels can be subject to several modes of regulation, going from posttranslational control to mRNA stability and transcriptional regulation [41-44]. The c-myc gene, although it contains a dual promoter, is predominantly transcribed from the P2 promoter [45]. It is shown to harbor a promoter-paused RNApol II [46] In addition to other important regulatory sites upstream of P2 promoter, an E2F binding site at position -58 from the P2 transcription start site has been identified [45,47]. This E2F site has been demonstrated to be essential for c-myc transcription. It negatively controls c-myc transcription by recruitment of pocket proteins such as p130 that will function as a scaffold for HDAC complexes [48]. Overlapping with the E2F site there is an element essential to remodel nucleosome structure and to open c-myc promoter [45].

c-Myc expression, like Id2 expression, is induced in two stages following PHx. The first induction during G0-G1 phase, takes place at the onset of liver regeneration and the second peak is observed during the transition into the $S$ phase. However, both peaks of c-myc expression are the result of different modes of regulation. While the first increase of c-myc expression is caused by a transcriptional and posttranscriptional up-regulation of the gene, the second peak results as a consequence of enhanced mRNA stability [49]. During G0-G1, cmyc transcriptional initiation increases at $1 \mathrm{~h}$ after PHx in mice liver. Strikingly, this transcrip- 
tional initiation has been shown to be compensated by a concomitant block of transcriptional elongation [46,49]. Elongation blockage of c-myc transcription has been considered as a mechanism to prevent over-expression of the mRNA [50].

The puzzling observation that both pausing and transcriptional initiation of c-myc are enhanced in the regenerating liver lead to hypothesize that increased transcriptional initiation of c-myc in this growth process might be driven by a component of a more general response [49]. Several genes that share common target sequences could be simultaneously activated by a common mechanism, but only additional and specific factors will render the final pattern of gene expression. Indeed, there are hundreds of E2F-target genes that, although transcriptionally dependent on RB proteins, do not show the same gene expression profile. This suggestive idea make us wonder about the identity of that "component of a more general response" involved in transcription initiation.
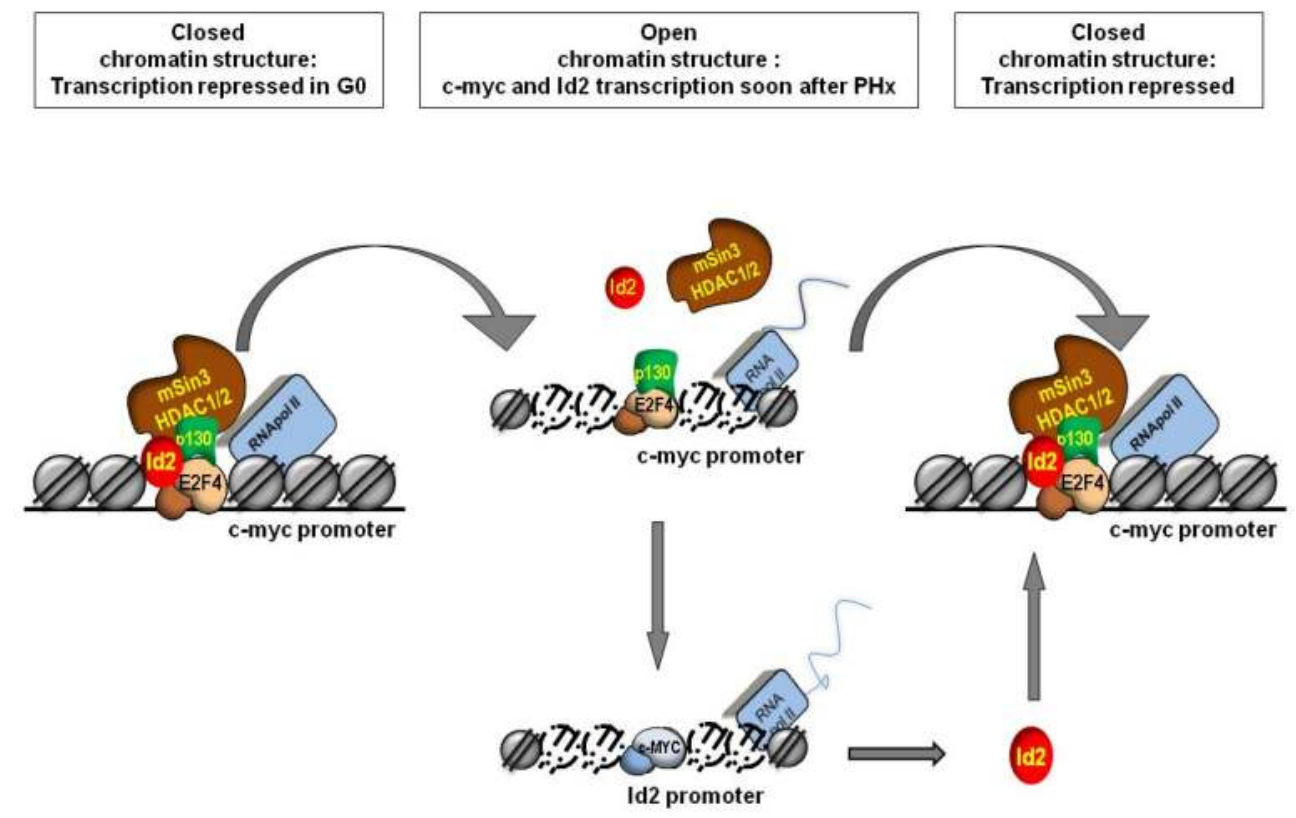

Figure 4. Model for the role of Id2 on c-myc expression in rat liver after PHx. In liver under basal conditions hepatocytes rest at G0. E2F4, p130, Id2 and the HDAC complex are bound on the c-myc promoter. RNApol II is paused on gene promoter. Since chromatin is deacetylated and closed, paused RNApol II cannot progress and c-myc transcription is repressed. Soon early after PHx, Id 2 and the HDAC complex are released. Chromatin will be acetylated and opened. This chromatin structure will allow paused RNApol II to progress. c-myc transcription will be transiently up-regulated. c-Myc protein will bind to Id 2 promoter inducing its expression. High levels of Id 2 will favor its reassemble on c-myc promoter together with the HDAC complex. Chromatin structure will be closed and RNApol II will remain paused on cmyc promoter. Consequently, c-myc expression is shut off.

Id2 could play such a role as part of a common mechanism for transcription of E2F-driven genes. Id2 has been shown to bind to a repressor complex on c-myc promoter that affects 
chromatin structure (figure 4) [30]. On quiescent hepatocytes Id2, mSin3A (a co-repressor that constitutes the core of HDAC1 and HDAC2 complex), E2F4 and p130 remain bound to the cmyc promoter repressing its expression. Additionally, immunoprecipitation experiments revealed that Id2 is bound to $\mathrm{mSin} 3 \mathrm{~A}(\mathrm{HDAC}), \mathrm{E} 2 \mathrm{~F} 4$ and p130. Upon stimulation after $\mathrm{PHx}$, $\mathrm{Id} 2$ and $\mathrm{mSin} 3 \mathrm{~A}(\mathrm{HDAC})$ are released from the c-myc promoter concurrently with a, at least in part c-myc transcriptional up-regulation. On the other hand, the transient c-myc upregulation is followed by an increase of Id 2 mRNA. Since c-Myc modulates Id 2 transcription and Id 2 modulates c-myc expression, a possible regulatory loop would finally control c-myc abundance. These observations lead to propose Id 2 as part of the priming events described for liver regeneration and perhaps for some other processes [30].

This hypothesis would go further from the initial idea of Id2 as a simple effector of c-myc to bypass the repressive activity of RB. In this sense, endogenous levels of activated Id 2 were shown to enhance but not to support the rate of proliferation in oligodendrocyte precursor cells at the time that slows the rate of differentiation [51]. The authors suggested that Id 2 takes part of an intrinsic timing mechanism to control or limit cell proliferation in biological processes highly dependent on a sequential chain of signals that must be transiently synthesized to coordinate the process.

The significance of the mechanism proposed for the role of Id2 in the regulation of c-myc expression, might be extended to the modulation of other genes. To ask whether Id 2 plays a global role on transcriptional regulation and/or if it is restricted to E2F4 target genes, we performed genome-wide ChIP-on-chip experiments with antibodies specific for mouse Id2 and E2F4 in quiescent mouse liver [manuscript in preparation]. Promoter arrays contained approximately 28,000 known mouse genes centered on the region from $-6 \mathrm{~kb}$ to $+2.5 \mathrm{~kb}$ relative to the transcription start site at an average resolution of $35 \mathrm{bp}$. Analysis of at least three independent experiments for each factor identified 871 target regions for Id2, and 9307 for E2F4. A comparison of Id2 and E2F4 target regions indicated a limited overlap of 550 target regions for both factors. Moreover, a genome-wide factor location analysis and region classification for E2F4 and Id2-bound regions compared to the genome revealed that although E2F4-alone showed a 100\% overlap with gene promoters, positive probes for E2F4/Id2 were partially overlapping with the first exon of genes. This observation suggests that there is a common structure in those genes modulated by E2F4/Id2 that differs from those bound by E2F4 alone. Moreover, E2F4 and Id2 seem to mutually influence each other's binding position and behave like a complex on chromatin. Finally, an in silico gene expression analysis for E2F4 or Id2 positive genes showed that Id2/E2F4-bound genes were mostly repressed in liver basal conditions versus those bound by E2F4 alone.

ChIP-on-chip data confirm that although not all E2F-target genes bind Id2, a subset of these genes might be modulated by the same mechanism as c-myc. This would be a promising molecular mechanism to explain the pleiotropic activities of Id2 affecting the expression of a variety of genes. Id2 could be one of those signals shared by a plethora of biological responses to different stimuli and part of a common mechanism for transcription initiation of different genes. 
Most of papers in the literature are focus on the role of Id2 in proliferating, undifferentiated or otherwise transformed cells. However, the behavior of Id2 seems to be quite different depending on the microenvironment, its expression levels, compartmentalization and/or phosphorylation. In other words, the role of Id2 in quiescent cells seems to be different from the one observed in long term proliferating cells. While "activated" Id2 seems to promote cell cycle progression after a proliferative stimulus, "inactivated" Id 2 seems to limit or to control the number of cell divisions in quiescent cells

\section{Signals involved in the molecular regulation of Id2 activity and function}

Id 2 expression levels have been classically described as a key event for Id 2 function. In general, Id 2 expression is high in proliferating cells and low or almost absent in non-proliferating cells such as terminally differentiated cells. However, Id 2 is a pleiotropic protein involved in many other functions different from cell proliferation. TGF- $\beta$ (a cytokine whose function is highly dependent on the microenvironment) represses Id2 expression in epithelial cells [34] but induces Id2 up-regulation in dendritic cells [52]. Glucocorticoids [53], starvation [54], antidiabetic agents and peroxisome proliferator-activated receptor (PPAR) gamma agonists or members of HDAC family including HDAC1-8 [9] are some of the many examples reported to inhibit Id 2 expression in specific cell types. Furthermore, this signals can be connected and coordinated. For instance, it seems that HDAC is involved in the modulation of Id2 expression through the interaction with several signaling pathways such as the HDAC/Ying Yang1, the Wnt- $\beta$-catenin-TCF7L2 and the BMP pathways that can be mutually coordinated in a different way in different cell types.

On the other hand, Id2 expression can be up-regulated by many factors such as glucose [55] insulin-like growth factor, estrogen, progesterone, thyroid hormone or hypoxia-inducible factor- $1 \alpha$ [review in ref 9]. Moreover, recently it has been published a large-scale RNAi screen to characterize genes involved in the regulation of Id2 expression [56]. To further complicate it, in addition to a growing list of up- and down-regulators of Id2 expression, the protein undergoes a rapid turnover, having a reported half-life of 20-60 min, depending on the cell type $[57,58]$. Thus, Id 2 can be regulated at several levels, i.e. transcriptional regulation and protein stability. Although the importance of these modes of regulation cannot be denied, they do not explain the underlying mechanisms for the regulation of Id2 activity. It is important to highlight that there are reports describing an important role for Id 2 in conditions in which its protein levels do not substantially change [51].

\subsection{Id2 phosphorylation and cell compartmentalization}

An important mechanism proposed to regulate Id 2 activity is the phosphorylation of specific residues. There is a phosphorylation site for CDK-type kinases close to the amino-terminal domain of Id2 proteins. This site comprises the consensus sequence for CDK substrates (S/T$\mathrm{P}-\mathrm{X}-\mathrm{K} / \mathrm{R})$ that is conserved among other members of the Id family. Id 2 has been shown to be phosphorylated in Ser5 within this consensus sequence by cyclin A/E/CDK2. This phosphor- 
ylation was reported to prevent Id2 binding to some bHLH factors and to block the activity of Id 2 in serum stimulated human fibroblasts. In vitro experiments with a mutant form of Id2 in which Ser5 was change to Ala (Id2-S5A), showed that when Id2 phosphorylation was blocked, Id2 was able to interact with the bHLH protein MyoD. In agreement with this, cyclinA/CDK2 phosphorylation of wild type Id2 prevented its binding to MyoD. The relation between cell proliferation and Id2 phosphorylation state was also established. Indeed, expression of Id2S5A caused a 50\% reduction in colony formation, suggesting that sustained unphosphorylated-Id2 is growth inhibitory [59]. Nevertheless, it seems that Id2 phosphorylation do not necessarily blocks Id2 interaction with other proteins. A latter report suggested that CDK2 phosphorylation of Ser5-Id2 is necessary for the interaction of Id2 with factors that allow nuclear transport such as E47 [60].

From these observations we should infer that Id2 phosphorylation at Ser5 does not block any of its possible interactions with other proteins. Interestingly,Id2 phosphorylated in vitro and in vivo was subject to a tryptic peptide mapping. The unique phosphopeptide found in vitro resulted to be phosphoSer5-Id2. However, two phosphopeptides were observed in the Id2 recovered from the experiment in vivo. Whereas the first phosphopeptide was also phosphoSer5-Id2, the identity of the second phosphopeptide in metabolically labeled Id2 needs to be determined. The authors suggested that it might reflect the action of a non-CDK kinase such as PKA or PKC on Id2 [59]. Although this possibility needs to be confirmed in vivo, these two kinases have been reported to phosphorylate Id2 in vitro [61]. In that case, it is reasonable to think that the different activities of Id2 or its interaction with other proteins could be dependent on the type or number of residues phosphorylated by specific kinases.

Another important consequence of Id2 phosphorylation on Ser5 has been pointed out above. This phosphorylation seems to be necessary for Id 2 nuclear localization and proliferating activity. Ectopic over-expression of Id2-S5A and wild type Id2 in smooth muscle cells show a different subcellular distribution. While wild type Id 2 localization was predominantly nuclear, mutant Id2-S5A was excluded from the nucleus,. Moreover, over-expression of wild type Id2 in smooth muscle cells leads to increase Id2 phosphorylation, down-regulation of p21 and enhanced cell proliferation. In contrast over-expression of unphosphorylated Id2-S5A could not promote cell proliferation [60].

The subcellular distribution is one of the major points for the regulation of Id2 activity and function. During differentiation of oligodendrocyte precursor cells (OPC) into oligodendrocytes, Id 2 protein levels are similar. Interestingly, the cellular distribution of Id 2 dramatically changed as OPC differentiate. Id2 was localized into the nucleus of undifferentiated OPC whereas in fully differentiated oligodendrocytes Id2 was localized in the cytoplasm [51]. Moreover, the authors show that Id2 needs to translocate from the nucleus to the cytosol prior to cell differentiation.

Id2 contains two export signals (NES1 and NES2) in the HLH domain and the C-terminal domain respectively. NES1 was found to be conserved among all members of the Id family, and NES2 was shown to be specific for Id2. Although Id2 transport between nucleus and cytosol had been proposed to be by passive diffusion, the recent data point out also to an active transport of Id2. Id 2 export from the nucleus is mediated by NES2. However, Id 2 does not 
contain a nuclear localization signal (NLS) and translocation from the cytosolic compartment into the nucleus should be mediated by its interaction with a NLS-containing protein. In this way, translocation can be mediated by interaction of Id2 with E proteins such as E47[60]. Phosphorylation of Id2 favors Id2/E47 interaction and nuclear translocation. Nevertheless, it is important to highlight that interaction with E proteins can also function sequestering Id2 in the cytosol [61]. Moreover, since Id 2 can bind to non-HLH proteins, other factors up-regulated during cell differentiation can sequester Id2 preventing its transport into the nucleus. A cytoskeleton-associated protein enigma homolog $(\mathrm{ENH})$ is a good example of a number of proteins described to retain Id2 in the cytosol. ENH is a member of the enigma family of PDZLIM domain proteins that is associated to the actin cytoskeleton by direct interaction between its PDZ domain and the $\alpha$-actinin. Id 2 binds to ENH through specific interaction between the ENH-LIM domain and the Id2-HLH domain. ENH, up-regulated during neural differentiation, sequesters Id 2 in the cytoplasm preventing cell-cycle progression and releasing bHLH factors from the restrictive activity of Id2 [62]

\subsection{Role of GSH on Id 2 modulation}

Id2 expression and interaction with other proteins has been shown to be modulated by GSH content $[63,64]$. The tripeptide glutathione is one of the most important systems against oxidative stress in the organism. Alteration of redox balance can affect cell signaling pathways through the induction of protein posttranslational modifications or, in an indirect manner, by the modulation of transcription factor binding activity and signal transduction pathways [65]. However, while the role of cell GSH content in the synthesis of DNA and protection against oxidative damage has been long established, little is known about its sub-cellular distribution and functions in specific compartments. Moreover, the nucleus of dividing cells dramatically change throughout progression into the cell-cycle. It has been recently shown that nuclear GSH content is high in proliferating cultured cells. On the contrary, quiescent cells show similar or lower GSH levels in the nucleus than in the cytosol [66]. In this sense, although most of data describe a significant increase in the hepatic GSH content at $12 \mathrm{~h}$ after PHx [67], at earlier times, when the priming events take place, there is a $49 \%$ reduction in GSH content [68]. Finally, an increasing number of evidences establish a correlation between GSH content and gene expression. Interestingly, it has been speculated that ROS generation induced by a shift in the redox status of cells could affect gene expression by altering chromatin configuration $[69,70]$.

\subsubsection{GSH depletion and Id2 up-regulation}

The nature of a rapid response triggered by a stimulus suggests pre-existing signals within the tissue that could modulate the expression of Id 2 and/or its activity. Moreover, the role of GSH as part of a sensitization process has been already described. GSH depletion sensitizes cells to c-myc-induced apoptosis or proliferation in a variety of cell types, such as myoblasts, melanoma cells or hepatocytes [65,71]. Treatment of rats with 1-buthionine-(S,R)-sulfoximine (BSO), the inhibitor of $\gamma$-glutamil-cysteine synthetase, induces a rapid GSH depletion in liver that in some way resembles the one observed after PHx (figure 5). Interestingly, BSO treatment also induces two marked peaks of c-myc and Id2 expression in rat liver [69]. The first peak of c- 
myc expression after GSH depletion shows a short up-regulation also described for other stimuli, a stereotypical transcriptional pulse that lasts for 2-3h and is followed by a shutoff [72]. The second peak of c-myc mRNA was shown to be the result of a posttranscriptional mRNA up-regulation.

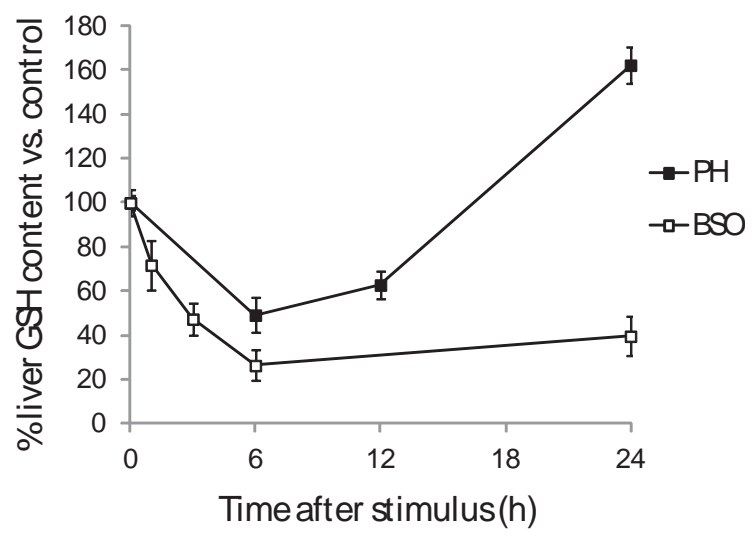

Figure 5. GSH content in rat liver after PHx or in BSO-treated animals. White squares represent GSH levels in rat liver during the time course of BSO experiment (Torres et al. refer 63) Black squares represent GSH levels in rat liver during the time course of liver regeneration after $\mathrm{PHx}$ (Lee et al. ref. 68).

GSH depletion seemed to be involved in the release of the repressor complex Id2/ $\mathrm{mSin} 3 \mathrm{~A}(\mathrm{HDAC})$ from the c-myc promoter as it occurred after PHx (figure 4). The proteins Id2 and $\mathrm{mSin} 3 \mathrm{~A}$ are released from the c-myc promoter as soon as the GSH content decreases $20 \%$ under basal levels. Concomitantly with the release of the repressor complex, c-myc expression is induced. When Id2 and mSin3A turns back to the c-myc promoter, the transcription of the gene is down-regulated. Nevertheless it could not be established a relationship between GSH content and the return of Id2 to the c-myc promoter. However, linking these observations to liver regeneration, it would be possible that a decrease in GSH content very early after $\mathrm{PH}$ could promote the release of $\mathrm{Id} 2 / \mathrm{mSin} 3 \mathrm{~A}(\mathrm{HDAC})$ from the c-myc promoter.

Nevertheless, this event would not be enough to induce the transient transcription initiation of c-myc observed after GSH depletion with BSO. However, the release of Id2 and $\mathrm{mSin} 3 \mathrm{~A}(\mathrm{HDAC})$ from the c-myc promoter would induce profound changes in chromatin structure to become accessible to transcription factors. Indeed, a deeper insight into the molecular mechanisms involved in the regulation of transcription at the first peak of c-myc expression after GSH depletion, suggested an important role for STAT3 at this peak. GSH depletion induces STAT3 phosphorylation, recruitment of CBP/p300 (HAT) and binding to a region that overlaps the E2F site in the c-myc gene P2 promoter shown to be important for nucleosomal structure [73]. These observations are in agreement with the idea of GSH and Id2 as part of a general mechanism to sensitize cells to respond to a given stimulus (figure 6): Id2 release would lead to an open chromatin structure allowing transcription factors to gain access 
to gene promoters. The specificity of the response would be achieve by the precise combination of activated-transcription factors and their binding to a particular gene promoter, like in this case occurs with STAT3 and c-myc promoter.

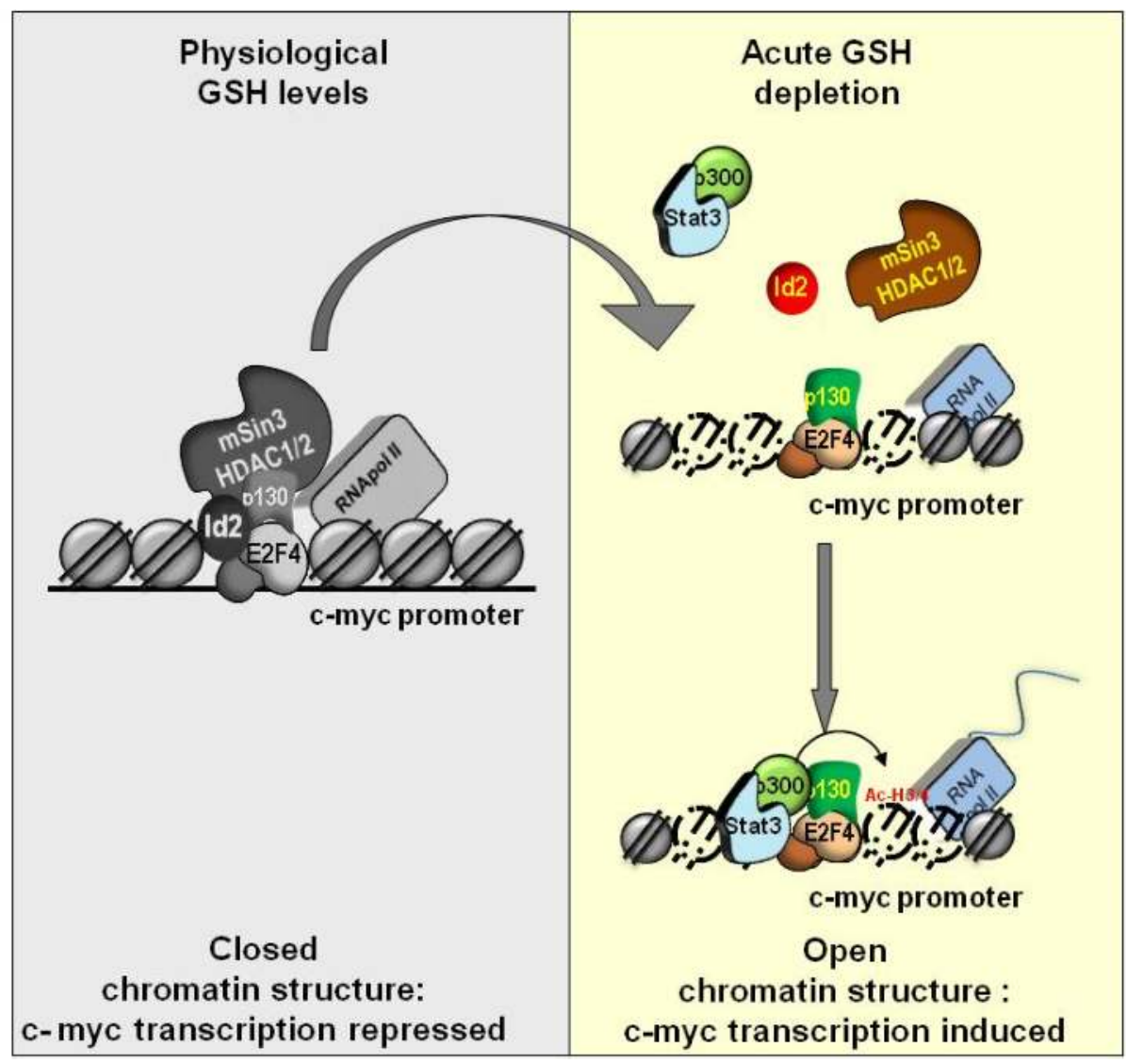

Figure 6. Model for the role of GSH on the modulation of Id2 activity. In quiescent cells, under physiological GSH content E2F4, p130, Id2 and the HDAC complex are bound on the c-myc promoter. Chromatin structure will be closed, inaccessible to transcription factors. RNApol II is paused on the c-myc promoter and unable to transcribe the gene. Early after an acute GSH depletion, Id 2 and HDAC would be released from c-myc promoter. Chromatin will be now open and accessible for transcription factors like STAT3 that could now specifically induce transcription initiation. These events would enhance an open hyperacetylated chromatin structure and RNApol II progression.

\subsubsection{GSH depletion and Id2 down-regulation}

It has been demonstrated that drugs, infections, inflammation, or cell proliferation can alter GSH levels and cause a shift in the GSH/GSSG redox status of cells [65]. Over-expression of cmyc and GSH depletion has been described in a variety of both experimentally induced and 
naturally occurring liver diseases including hepatocarcinoma, HCV infection, liver cirrhosis, apoptosis and drug-induced toxicity [74]. The information about the signals mediating the induction or repression of Id2 transcription in response to different stimuli is scarce and sometimes contradictory. GSH depletion by BSO has been shown to induce Id 2 expression by c-Myc [63]. Conversely, TGF- $\beta$ which decreases the concentration of GSH in a variety of cell types [75], has been shown to induce Id2 down-regulation through the modulation of c-Myc [34]. Id2 is always defined as a pleiotropic protein whose behavior depends on its expression levels, cell type, stimulus and in general on the cell microenvironment. In addition, Id2 is a downstream target of multiple signaling pathways that can converge or interact with each other giving rise to a specific response. Taking into account all these considerations, it is not surprising that under pathological conditions the effect of GSH depletion on Id2 regulation could be quite different.

Acetaminophen (APAP)-induced intoxication, the main cause of drug-induced liver failure in the United States and Great Britain, produces a dramatic GSH depletion in the liver. The deleterious effect of APAP excess has been attributed to its metabolization by the cytochrome P450 system, which gives rise to the formation of N-acetyl-p-benzoquinoneimine (NAPQI). This derivative of APAP will then react with GSH, inducing its rapid depletion within the liver and generating oxidative stress. Moreover, when the formation of NAPQI exceeds the GSH content, NAPQI will covalently bind to cellular proteins. Therefore, the hepatotoxicity of APAP overdose is the consequence of the additive effect of NAPQI formation, GSH depletion, oxidative stress and the generation of protein adducts (figure 7) [76].

Concomitantly with the GSH depletion there is a dramatic transcriptional-dependent decrease of Id2 expression in response to APAP toxicity [64]. RNApol-II is released from Id 2 coding region and, the promoter is hypoacetylated. These data might suggest that the combination of GSH depletion, oxidative stress and APAP derivatives that are produced after APAP intoxication (depicted in figure 7) may be responsible for the observed Id2 repression.

Id2 expression is known to be dependent on c-Myc binding to its promoter $[19,20]$, but surprisingly GSH depletion stimulated by APAP-overdose increases c-myc transcription. However, the mRNA steady state levels do not correlate with the protein levels and consistently, c-Myc does not bind to Id2 promoter in response to APAP-overdose. Although APAP induces c-myc transcription, it also triggers the proteasome pathway leading to decreased cMyc half life, which is in agreement with other observations showing the APAP-induced degradation of specific proteins $[77,78]$. On the other hand, it has been shown that c-Myc downregulation is involved in the Id2 repression [34]. Accordingly, a decreased in c-Myc half life induced by APAP overload would lead to Id2 repression.

Data from these experiments support the idea of a regulatory loop between Id2 and c-myc: The down-regulation of Id 2 would favor the release of the repressor complex mSin3A(HDAC) from c-myc promoter and would render a peak of c-myc expression.

On the other hand, although APAP-overdose induces c-myc mRNA up-regulation, it decreases c-Myc protein half life and therefore protein levels are diminished. 


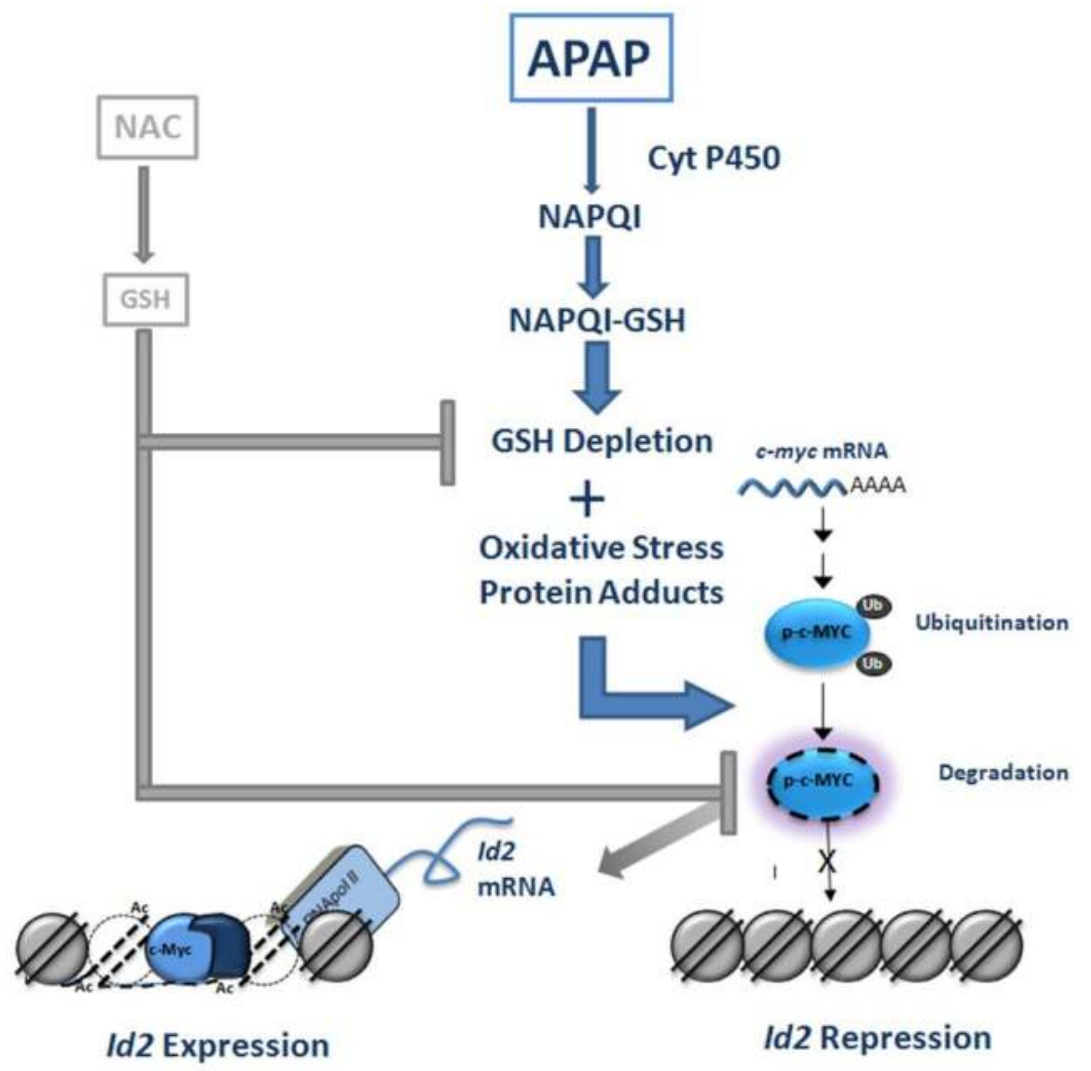

Figure 7. Model for the regulation of Id2 expression in rat liver after acetaminophen overdose and protection by $\mathrm{N}$ acetylcysteine. APAP-overdose is metabolized by the cytochrome P450 system giving rise to the highly reactive derivative NAPQI. This derivative will be detoxified by covalent binding to GSH, which will be soon depleted inducing c-myc expression. When NAPQI exceeds GSH stores, oxidative stress and protein adducts formation will induce c-Myc degradation. The absence of c-Myc from Id 2 promoter will block Id2 basal expression. NAC replenishes GSH stores thus preventing GSH depletion, protein adduct formation and the proteasome pathway activation. In the presence of NAC, cMyc degradation is prevented. Therefore, c-Myc binds to Id 2 promoter supporting its basal expression.

Administration of N-acetycysteine (NAC) prior to APAP-overload prevents GSH depletion. Moreover, it has been suggested that NAC interferes with the proteasome pathway induced by APAP toxicity. NAC inhibits the $26 \mathrm{~S}$ proteasome activity in cultured cells increasing the stability of $\mathrm{I} \kappa \mathrm{B} \alpha$ and $\mathrm{p} 53$ [79]. Furthermore, the proteasome has been described as a redoxsensitive system [80]. Interestingly, NAC pretreatment prevents c-Myc degradation and accordingly, RNApol-II release from Id2 coding region is also prevented. Consistently, Id2 promoter is hyperacetylated and Id2 repression prevented. It is noteworthy to mention that there is a parallelism between NAC protection and keeping Id2 expression at baseline levels. 
Covalent binding of NAPQI to mitochondrial proteins has been shown to induce mitochondrial dysfunction and to enhance the formation of reactive oxygen species. The effective protection of high doses of NAC on APAP-induced liver damage has been attributed to the recovery of hepatic and mitochondrial GSH levels. Nevertheless, NAC is not only a GSH precursor, but it is also a reducing and antioxidant agent acting as a direct scavenger of free radicals. Altogether this data suggest that NAC by itself and/or GSH seem to improve the efficiency of the detoxifying system preventing, among other events, Id 2 down-regulation. But more importantly, the effect of GSH and oxidative stress in the modulation of Id 2 in the context of a wide spread pathology such as the intoxication by APAP seems to be the result of cumulative and converging pathways. These observations highlight the importance of establishing the correct landscape to study the role of Id2, the molecular mechanisms governing its functions, and the physiological and pathological consequences of its regulation and deregulation.

\section{Conclusions and perspectives}

Among the four members of Id family of proteins, Id2 is a key player in the modulation of cell proliferation. However, many questions about its function in vivo remain unresolved. Most of data about the role of Id 2 as a proliferating factor are referred to either tumors or cultured and/or transformed cells that might reflect conditions far away from a physiological situation. This is especially relevant since it is well known and generally accepted that the modulation of Id2 activity and its many functions depend on the cell microenvironment. We already discussed the diametrically opposing effects of GSH depletion on Id2 in a pathological condition such as APAP-induced toxicity and in an experimental condition of BSO-induced GHS depletion [63,64].

In addition, the functions of Id2 are not exclusively dependent on its abundance, but also on its posttranslational modifications, sub-cellular distribution and its interaction with a number of different proteins [5,6,11-15]. Nevertheless, most studies use ectopic over-expression of Id2 as a tool to determine its functions and molecular mechanisms with independence of its microenvironment. To understand the role of Id 2 during cell proliferation, we should take into account that the enzymatic activities, signaling networks and redox status of cells dramatically change throughout the different phases of the cell cycle. A static image of Id 2 during cell cycle would lead to confusion. Id 2 gene expression is exposed to a tight control, restrained by a precise timing and coordinated with the expression of other proteins to interact with (i.e. ENH is induced during cell differentiation to sequester Id2 within the cytoplasm).

Following this rational, we can deduce that Id2 functions will change as the cell progress through the cell cycle. Actually, Id2 shows two marked peaks of gene expression during G0G1 and G1-S respectively that will have different effects on the regulation of the cell cycle. In quiescent cells Id2 binds to hypophosphorylated p130, E2F4 and a co-repressor complex. Id2 expression is low at this stage. The role of Id 2 at this phase is most probably to limit the number of cell divisions enhancing repression of E2F-target genes. 
During G0-G1 transition, at the onset of the mitogenic stimuli there is a rapid change in both, cytosolic and nuclear compartments (i.e. redox status, signaling networks, enzymatic activities) that will change the preferential Id2-p130 interaction. Initial studies suggested that HDAC complexes directly bound to RB proteins. Nevertheless, subsequent studies revealed that recruitment of HDAC complexes to RB was mediated by RBP1 [28]. As already discussed, the release of $R B$ restrictive activity is the result of cumulative CDK-mediated phosphorylations. These phosphorylations do not have an effect exclusively on RB proteins, but also on RBP1 that bridges SAP30/mSin3(HDAC) to RB proteins. CDK-mediated phosphorylation of RBP1 induces its dissociation from RB [28]. It seems that during G1 phase the multiple and cumulative CDK-mediated phosphorylations of cell cycle-dependant proteins would set a threshold. This ensures that the G1 phase can only be overcome when CDKs reach a critical phosphorylation threshold for efficient dissociation of HDAC and RB from E2F. Therefore it is not a matter of "all or nothing", but the binding affinities between co-repressors, E2F and RB gradually change. Following the same rational, it is tempted to speculate that Id2 could remain bound to the repressor complex SAP30/mSin3/HDAC in quiescent cells and upon a stimulus, a CDKmediated phosphorylation of Id 2 or its interacting proteins would release Id 2 and the repressor complex from p130. As already referred, Id2 is known to be phosphorylated during G1 phase by cyclin $\mathrm{A} / \mathrm{CDK} 2$ and cyclin E/CDK2 [24,59]. This phosphorylation may target Id2 for ubiquitn-mediated proteasomal degradation and/or change its affinity for specific bHLH proteins $[81,82]$. Alternatively, other posttranslational modifications of Id2 or Id2-interacting proteins might condition Id2 release from E2F4/5 and p130 during G0-G1 transition. In the future it will be crucial to determine those signals that modulating Id2-binding activities can condition the transition from G0 to G1.

It is important to highlight that independently of the molecular mechanism that trigger Id2 release, this event seems to have a profound effect on chromatin structure changing its accessibility to specific transcription factors that on their behalf could recruit HATs (as depicted in figure 6). This mechanism seems to operate not only in a small subset of genes, but on the contrary it can be extended to a larger group of genes (we have observed almost 400 Id2/E2F4-bound genes in our ChIP-on-chip experiments). We propose here the suggestive hypothesis that Id 2 could take part of a common mechanism for cell sensitization or priming to fully respond to a mitogenic stimulus inducing an open chromatin structure. The resultant expression of a particular gene will depend on the activation and DNA-binding of the precise combination of transcription factors for the specific gene. Nevertheless, this hypothesis remains as a challenge to cover and understand the molecular mechanisms of Id2 regulation and more importantly to unveil Id2 role during G0-G1 transition.

\section{Acknowledgements}

This work was supported in part by grants from Ministerio de Ciencia e Innovación (FIS PS09-02360) to E.R.G-T and Plan Nacional I+D+I (BFU2010-18253) and Generalitat Valenciana (PROMETEO 2010-075) to J.R.V. 


\section{Author details}

Elena R. García-Trevijano, Luis Torres, Rosa Zaragozá and Juan R. Viña

*Address all correspondence to: elena.ruiz@uv.es

Department of Biochemistry and Molecular Biology, University of Valencia, Valencia, Spain

\section{References}

[1] Michalopoulos GK Liver regenerationJ. Cell Physiol.(2007). , 213, 286-300.

[2] Leist, M, Gantner, F, Bohlinger, I, Germann, P. G, Tiegs, G, \& Wendel, A. Murine hepatocyte apoptosis induced in vitro and in vivo by TNF-alpha requires transcriptional arrest. J Immunol.(1994). , 153, 1778-1788.

[3] Benezra, R, Davis, R. L, Lockshon, D, Turner, D. L, \& Weintraub, H. The protein Id: a negative regulator of helix-loop-helix DNA binding proteins. Cell. (1990). , 61(1), 49-59.

[4] Murre, C, Mccaw, P. S, Vaessin, H, Caudy, M, Jan, L. Y, Jan, Y. N, Cabrera, C. V, Buskin, J. N, Hauschka, S. D, Lassar, A. B, et al. Interactions between heterologous helixloop-helix proteins generate complexes that bind specifically to a common DNA sequence. Cell. (1989). , 58(3), 537-44.

[5] Massari, M. E, \& Murre, C. Helix-loop-helix proteins: regulators of transcription in eucaryotic organisms. Mol Cell Biol. (2000). , 20, 429-440.

[6] Uzinova, M. B, \& Benezra, R. Id proteins in development, cell cycle and cancer. Trends Cell Biol. (2003). , 13(8), 410-8.

[7] Kim, H. J, Hong, J. M, Yoon, K. A, Kim, N, Cho, D. W, Choi, J. Y, Lee, I. K, \& Kim, S. $\mathrm{Y}$. Early growth response 2 negatively modulates osteoclast differentiation through upregulation of Id helix-loop-helix proteins. Bone. (2012). , 51(4), 643-50.

[8] Yokota, Y, Mansouri, A, Mori, S, Sugawara, S, Adachi, S, Nishikawa, S, \& Gruss, P. Development of peripheral lymphoid organs and natural killer cells depends on the helix-loop-helix inhibitor Id2. Nature. (1999). , 397(6721), 702-6.

[9] Chen, X. S, Zhang, Y. H, Cai, Q. Y, \& Yao, Z. X. ID2: A negative transcription factor regulating oligodendroglia differentiation. J Neurosci Res. (2012). , 90(5), 925-32.

[10] Iavarone, A, King, E. R, Dai, X. M, Leone, G, Stanley, E. R, \& Lasorella, A. Retinoblastoma promotes definitive erythropoiesis by repressing Id 2 in fetal liver macrophages. Nature. (2004). , 432(7020), 1040-5. 
[11] Norton, J. D, \& Helix-loop, I. D. helix proteins in cell growth, differentiation and tumorigenesis J. Cell Sci. (2000). , 113, 3897-3905.

[12] Lasorella, A, Uo, T, \& Iavarone, A. Id proteins at the cross-road of development and cancer. Oncogene. (2001). , 20, 8326-8333.

[13] Zebedee, Z, \& Hara, E. Id proteins in cell cycle control and cellular senescence Oncogene. (2001). , 20, 8317-8325.

[14] Yokota, Y, \& Mori, S. Role of Id family proteins in growth control J. Cell. Physiol. (2002). , 190, 21-28.

[15] Fong, S, Debs, R. J, \& Desprez, P. Y. Id genes and proteins as promising targets in cancer therapy.Trends Mol Med. (2004). , 10(8), 387-92.

[16] Coma, S, Amin, D. N, Shimizu, A, Lasorella, A, Iavarone, A, \& Klagsbrun, M. Id2 promotes tumor cell migration and invasion through transcriptional repression of semaphorin 3F. Cancer Res. (2010). , 70(9), 3823-32.

[17] Iavarone, A, Garg, P, Lasorella, A, Hsu, J, \& Israel, M. A. The helix-loop-helix protein Id-2 enhances cell proliferation and binds to the retinoblastoma protein. Genes Dev. (1994). , 8(11), 1270-84.

[18] Norton, J. D, \& Atherton, G. T. Coupling of cell growth control and apoptosis functions of Id proteins. Mol Cell Biol. (1998). , 18(4), 2371-81.

[19] Lasorella, A, Boldrini, R, Dominici, C, Donfrancesco, A, Yokota, Y, Inserra, A, \& Iavarone, A. Id2 is critical for cellular proliferation and is the oncogenic effector of $\mathrm{N}$ myc in human neuroblastoma. Cancer Res. (2002). , 62(1), 301-6.

[20] Lasorella, A, Noseda, M, Beyna, M, \& Iavarone, A. Id2 is a retinoblastoma protein target and mediates signalling by Myc oncoproteins. Nature. (2000). , 407(6804), 592-8.

[21] Maruyama, H, Kleeff, J, Wildi, S, Friess, H, Büchler, M. W, Israel, M. A, \& Korc, M. Id-1 and Id-2 are overexpressed in pancreatic cancer and in dysplastic lesions in chronic pancreatitis. Am J Pathol. (1999). , 155(3), 815-22.

[22] Gray, M. J, Dallas, N. A, Van Buren, G, Xia, L, Yang, A. D, Somcio, R. J, Gaur, P, Mangala, L. S, Vivas-mejia, P. E, Fan, F, Sanguino, A. M, Gallick, G. E, Lopez-berestein, G, Sood, A. K, \& Ellis, L. M. Therapeutic targeting of Id2 reduces growth of human colorectal carcinoma in the murine liver. Oncogene. (2008). , 27(57), 7192-200.

[23] Hasskarl, J, \& Münger, K. Id proteins--tumor markers or oncogenes? Cancer Biol Ther. (2002). , 1(2), 91-6.

[24] Matsumura, M. E, Lobe, D. R, \& Mcnamara, C. A. Contribution of the helix-loop-helix factor Id2 to regulation of vascular smooth muscle cell proliferation. J Biol Chem. (2002). , 277(9), 7293-7. 
[25] Rothschild, G, Zhao, X, Iavarone, A, \& Lasorella, A. E Proteins and Id2 converge on to regulate cell cycle in neural cells. Mol Cell Biol. (2006). , 57Kip2.

[26] Lasorella, A, Iavarone, A, \& Israel, M. A. Id2 specifically alters regulation of the cell cycle by tumor suppressor proteins. Mol Cell Biol. (1996). , 16(6), 2570-8.

[27] Chen, H. Z, Tsai, S. Y, \& Leone, G. Emerging roles of E2Fs in cancer: an exit from cell cycle control. Nat Rev Cancer. (2009). , 9(11), 785-97.

[28] Suryadinata, R, Sadowski, M, Steel, R, \& Sarcevic, B. Cyclin-dependent kinase-mediated phosphorylation of $\mathrm{RBP} 1$ and $\mathrm{pRb}$ promotes their dissociation to mediate release of the SAP30 mSin3 HDAC transcriptional repressor complex.J Biol Chem. (2011). , 286(7), 5108-18.

[29] Fan, L. X, Li, X, Magenheimer, B, \& Calvet, J. P. Li X Inhibition of histone deacetylases targets the transcription regulator Id2 to attenuate cystic epithelial cell proliferation. Kidney Int. (2012). , 81(1), 76-85.

[30] Rodríguez, J. L, Sandoval, J, Serviddio, G, Sastre, J, Morante, M, Perrelli, M. G, Martínez-chantar, M. L, Viña, J, Viña, J. R, Mato, J. M, Avila, M. A, Franco, L, López-rodas, G, \& Torres, L. Id2 leaves the chromatin of the E2F4-c-myc promoter during hepatocyte priming for liver regeneration. Biochem J. (2006). , 130.

[31] Zhang, J. M, Zhao, X, Wei, Q, \& Paterson, B. M. Direct inhibition of G(1) cdk kinase activity by MyoD promotes myoblast cell cycle withdrawal and terminal differentiation. EMBO J. (1999). , 18(24), 6983-93.

[32] Roberts, E. C, Deed, R. W, Inoue, T, \& Norton, J. D. Sharrocks AD Id helix-loop-helix proteins antagonize pax transcription factor activity by inhibiting DNA binding. Mol Cell Biol. (2001). , 21(2), 524-33.

[33] Stinson, J, Inoue, T, Yates, P, Clancy, A, Norton, J. D, \& Sharrocks, A. D. Regulation of TCF ETS-domain transcription factors by helix-loop-helix motifs. Nucleic Acids Res. (2003). , 31(16), 4717-28.

[34] Siegel, P. M, Shu, W, \& Massagué, J. Mad upregulation and Id2 repression accompany transforming growth factor (TGF)-beta-mediated epithelial cell growth suppression. J Biol Chem. (2003). , 278(37), 35444-50.

[35] Morrow, M. A, Mayer, E. W, Perez, C. A, Adlam, M, \& Siu, G. Overexpression of the Helix-Loop-Helix protein Id2 blocks T cell development at multiple stages. Mol Immunol. (1999). , 36(8), 491-503.

[36] Vandeputte, D. A, Troost, D, Leenstra, S, Ijlst-keizers, H, Ramkema, M, Bosch, D. A, Baas, F, Das, N. K, \& Aronica, E. Expression and distribution of id helix-loop-helix proteins in human astrocytic tumors. Glia. (2002). , 38(4), 329-38. 
[37] Cao, Y, Liu, X, Zhang, W, Deng, X, Zhang, H, Liu, Y, Chen, L, \& Thompson, E. A. Townsend CM Jr, Ko TC. TGF-beta repression of Id2 induces apoptosis in gut epithelial cells. Oncogene. (2009). , 28, 1089-1098.

[38] Kim, N. S, Kim, H. T, Kwon, M. C, Choi, S. W, Kim, Y. Y, Yoon, K. J, Koo, B. K, Kong, M. P, Shin, J, \& Cho, Y. Kong YY Survival and differentiation of mammary epithelial cells in mammary gland development require nuclear retention of Id2 due to RANK signaling. Mol Cell Biol. (2011). , 31(23), 4775-88.

[39] Prisco, M, Peruzzi, F, Belletti, B, \& Baserga, R. Regulation of Id gene expression by type I insulin-like growth factor: roles of Stat 3 and the tyrosine 950 residue of the receptor. Mo.1 Cell. Biol. (2001). , 21, 5447-5458.

[40] Oster, S. K, Ho, C. S, Soucie, E. L, \& Penn, L. Z. The myc oncogene: MarvelouslY Complex. Adv Cancer Res. (2002). , 84, 81-154.

[41] Vervoorts, J, Lüscher-firzlaff, J, \& Lüscher, B. The ins and outs of MYC regulation by posttranslational mechanisms. J Biol Chem. (2006). , 281, 34725-3479.

[42] Langa, F, Lafon, I, Vandormael-pournin, S, Vidaud, M, Babinet, C, \& Morello, D. Healthy mice with an altered c-myc gene: role of the 3 ' untranslated region revisited..Oncogene. (2001). , 20, 4344-4353.

[43] Levens, L. D. Making Myc. Curr. Top. Microbiol. Immunol., (2006). , 302, 1-32.

[44] Thomas, L. R, \& Tansey, W. P. Proteolytic control of the oncoprotein transcription factor Myc. Adv. Cancer Res. (2011). , 110, 77-106.

[45] Albert, T, Wells, J, Funk, J. O, Pullner, A, Raschke, E. E, Stelzer, G, Meisterernst, M, Farnham, P. J, \& Eick, D. The chromatin structure of the dual c-myc promoter P2 is regulated by separate elements. J Biol Chem. (2001). , 1.

[46] Bentley, D. L, \& Groudine, M. A block to elongation is largely responsible for decreased transcription of c-myc in differentiated HL60 cells. Nature. (1986). , 321, 702-6.

[47] Hamel, P. A, Gill, R. M, Phillips, R. A, \& Gallie, B. L. Transcriptional repression of the E2-containing promoters EIIaE, c-myc, and RB1 by the product of the RB1 gene. Mol Cell Biol. (1992). , 12, 3431-3438.

[48] Iavarone, A, \& Massagué, J. E. F and histone deacetylase mediate transforming growth factor beta repression of cdc25A during keratinocyte cell cycle arrest. Mol. Cell. Biol. (1999). , 19, 916-922.

[49] Morello, D, Fitzgerald, M. J, Babinet, C, \& Fausto, N. c-m. y. c. c-fos, and c-jun regulation in the regenerating livers of normal and $\mathrm{H}-2 \mathrm{~K} / \mathrm{c}-\mathrm{myc}$ transgenic mice. Mol Cell Biol. (1990). , 10(6), 3185-93.

[50] Eick, D, \& Bornkamm, G W. Transcriptional arrest within the first exon is a fast control mechanism in c-myc gene expression. Nucleic Acids Res.(1986). , 14, 8331-8346. 
[51] Wang, S, Sdrulla, A, Johnson, J. E, Yokota, Y, \& Barres, B. A. A role for the helix-loophelix protein Id2 in the control of oligodendrocyte development. Neuron. (2001). , 29(3), 603-14.

[52] Hacker, C, Kirsch, R. D, Ju, X. S, Hieronymus, T, Gust, T. C, Kuhl, C, Jorgas, T, Kurz, S. M, Rose-john, S, Yokota, Y, \& Zenke, M. Transcriptional profiling identifies Id2 function in dendritic cell development.Nat Immunol. (2003). , 4(4), 380-6.

[53] Zilberfarb, V, Siquier, K, Strosberg, A. D, \& Issad, T. Effect of dexamethasone on adipocyte differentiation markers and tumour necrosis factor-alpha expression in human PAZ6 cells. Diabetologia. (2001). , 44(3), 377-86.

[54] González Mdel CCorton JC, Acero N, Muñoz-Mingarro D, Quirós Y, Alvarez-Millán JJ, Herrera E, Bocos C Peroxisome proliferator-activated receptor $\alpha$ agonists differentially regulate inhibitor of DNA binding expression in rodents and human cells. PPAR Res. (2012).

[55] Grønning, L. M, Tingsabadh, R, Hardy, K, Dalen, K. T, Jat, P. S, Gnudi, L, \& Shepherd, P. R. Glucose induces increases in levels of the transcriptional repressor Id2 via the hexosamine pathway.Am J Physiol Endocrinol Metab. (2006). E, 599-606.

[56] Wu, N, Castel, D, Debily, M. A, Vigano, M. A, Alibert, O, Mantovani, R, Iljin, K, Romeo, P. H, \& Gidrol, X. Large scale RNAi screen reveals that the inhibitor of DNA binding 2 (ID2) protein is repressed by family member p63 and functions in human keratinocyte differentiation. J Biol Chem. (2011). , 53.

[57] Deed, R. W, Armitage, S, Brown, M, \& Norton, J. D. Regulation of Id-HLH transcription factor function in third messenger signalling. Biochem Soc Trans. (1996). S.

[58] Bounpheng, M. A, Dimas, J. J, Dodds, S. G, \& Christy, B. A. Degradation of Id proteins by the ubiquitin-proteasome pathway.FASEB J. (1999). , 13(15), 2257-64.

[59] Hara, E, Hall, M, \& Peters, G. Cdk2-dependent phosphorylation of Id2 modulates activity of E2A-related transcription factors. EMBO J. (1997). , 16(2), 332-42.

[60] Matsumura, M. E, Lobe, D. R, \& Mcnamara, C. A. Contribution of the helix-loop-helix factor Id2 to regulation of vascular smooth muscle cell proliferation. J Biol Chem. (2002). , 277(9), 7293-7.

[61] Samanta, J, \& Kessler, J. A. Interactions between ID and OLIG proteins mediate the inhibitory effects of BMP4 on oligodendroglial differentiation Development. (2004). , 131(17), 4131-42.

[62] Lasorella, A. Iavarone A The protein ENH is a cytoplasmic sequestration factor for Id2 in normal and tumor cells from the nervous system Proc Natl Acad Sci U S A. (2006). , 103(13), 4976-81. 
[63] Torres, L, Sandoval, J, Penella, E, Zaragozá, R, García, C, Rodríguez, J. L, Viña, J. R, \& García-trevijano, E. R. In vivo GSH depletion induces c-myc expression by modulation of chromatin protein complexes.Free Radic Biol Med. (2009). , 46(11), 1534-42.

[64] Penella, E, Sandoval, J, Zaragozá, R, García, C, Viña, J. R, Torres, L, \& García-trevijano, E. R. Molecular mechanisms of Id2 down-regulation in rat liver after acetaminophen overdose. Protection by N-acetyl-L-cysteine. Free Radic Res. (2010). , 44(9), 1044-53.

[65] Han, D, Hanawa, N, Saberi, B, \& Kaplowitz, N. Mechanisms of liver injury. III. Role of glutathione redox status in liver injury. Am. J. Physiol. Gastrointest. Liver Physiol. (2006). G, 1-7.

[66] Diaz Vivancos PWolff T, Markovic J, Pallardó FV, Foyer CH.A nuclear glutathione cycle within the cell cycle. Biochem J. (2010). , 431(2), 169-78.

[67] Huang, Z. Z, Li, H, Cai, J, Kuhlenkamp, J, Kaplowitz, N, \& Lu, S. C. Changes in glutathione homeostasis during liver regeneration in the rat. Hepatology. (1998). , 27, 147-53.

[68] Lee, S. J, \& Boyer, T. D. The effect of hepatic regeneration on the expression of the glutathione S-transferases. Biochem. J. (1993). , 293, 137-142.

[69] Hitchler, M. J, \& Domann, F. E. An epigenetic perspective on the free radical theory of development. Free Radic Biol Med. (2007). , 43, 1023-36.

[70] Hitchler, M. J, \& Domann, F. E. Redox regulation of the epigenetic landscape in Cancer: A role for metabolic reprogramming in remodeling the epigenome. Free Radic Biol Med. (2012).

[71] Biroccio, A, Benassi, B, Filomeni, G, Amodei, S, Marchini, S, Chiorino, G, Rotilio, G, Zupi, G, \& Ciriolo, M. R. Glutathione influences c-Myc-induced apoptosis in M14 human melanoma cells. J Biol Chem.(2002). , 277, 43763-43770.

[72] Levens, D. How the c-myc promoter works and why it sometimes does not. J Natl Cancer Inst Monogr. (2008). , 39, 41-43.

[73] Kiuchi, N, Nakajima, K, Ichiba, M, Fukada, T, Narimatsu, M, Mizuno, K, Hibi, M, \& Hirano, T. STAT3 is required for the gp130-mediated full activation of the c-myc gene. J Exp Med. (1999). , 189, 63-73.

[74] Chan, K. L, Guan, X. Y, \& Ng, I. O. High-throughput tissue microarray analysis of cmyc activation in chronic liver diseases and hepatocellular carcinoma. Hum Pathol. (2004). , 35, 1324-1331.

[75] Liu, R. M. Gaston Pravia KA.Oxidative stress and glutathione in TGF-beta-mediated fibrogenesis. Free Radic Biol Med. (2010). , 48(1), 1-15. 
[76] Jaeschke, H, Mcgill, M. R, \& Ramachandran, A. Oxidant stress, mitochondria, and cell death mechanisms in drug-induced liver injury: lessons learned from acetaminophen hepatotoxicity. Drug Metab Rev. (2012). , 44(1), 88-106.

[77] Lee, Y-S, Wan, J, Kim, B. J, Bae, M-A, \& Song, B. J. Ubiquitin-dependent degradation of protein despite phosphorylation at its $\mathrm{N}$ terminus by acetaminophen. J Pharm Exp Ther (2006). , 53.

[78] Abdelmegeed, M. A, Moon, K-H, Chen, C, Gonzalez, F. J, \& Song, B-J. Role of cytochrome $\mathrm{E} 1$ in protein nitration and ubiquitin-mediated degradation during acetaminophen toxicity. Biochem Pharmacol. (2010). , 450.

[79] Pajonk, F, Riess, K, \& Sommer, A. McBride W.H. N-acetyl-L-cysteine inhibits $26 \mathrm{~S}$ proteasome function: implications for effects on NF-kappaB activation. Free Rad Biol Med. (2002). , 32, 536-543.

[80] Breusing, N, \& Grune, T. Regulation of proteasome-mediated protein degradation during oxidative stress and aging. Biol Chem. (2008). , 389, 203-209.

[81] Lasorella, A, Stegmüller, J, Guardavaccaro, D, Liu, G, Carro, M. S, Rothschild, G, De La Torre-ubieta, L, Pagano, M, Bonni, A, \& Iavarone, A. Degradation of Id2 by the anaphase-promoting complex couples cell cycle exit and axonal growth. Nature. (2006). , 442, 471-474.

[82] Williams, S. A, Maecker, H. L, French, D. M, Liu, J, Gregg, A, Silverstein, L. B, Cao, T. C, Carano, R. A, \& Dixit, V. M. USP1 deubiquitinates ID proteins to preserve a mesenchymal stem cell program in osteosarcoma. Cell. (2011). , 146(6), 918-30. 